\title{
Exploration of Exosomal Micro RNA Biomarkers Related to Epithelial-to-Mesenchymal Transition in Pancreatic Cancer
}

\author{
FUMINORI SONOHARA, SUGURU YAMADA, SHIGEOMI TAKEDA, \\ MASAMICHI HAYASHI, MASAYA SUENAGA, YUKI SUNAGAWA, MITSURU TASHIRO, \\ HIDEKI TAKAMI, MITSURO KANDA, CHIE TANAKA, DAISUKE KOBAYASHI, \\ GORO NAKAYAMA, MASAHIKO KOIKE, MICHITAKA FUJIWARA and YASUHIRO KODERA
}

Department of Gastroenterological Surgery (Surgery II), Nagoya University Graduate School of Medicine, Nagoya, Japan

\begin{abstract}
Background/Aim: Epithelial-to-mesenchymal transition (EMT) plays important roles in cancer progression. This study aimed to identify the exosomal miRNA (exomiRNA) profiles related to the EMT status in pancreatic cancer (PC). Materials and Methods: Comprehensive exomiRNA-expression profiles in the culture media of PC cell lines were analyzed through microarray technology. The identified miRNAs were analyzed to investigate their clinical implication using The Cancer Genome Atlas (TCGA) dataset and clinical samples. Results: We prioritized 291 exo-miRNAs differentially expressed between epithelial and mesenchymal cell types. Among them, survival analysis based on the TCGA dataset revealed that mir-196b and mir-204 significantly stratify the prognosis of PC cases. In addition, analysis of cell lines indicated miR-196b-3p as a mesenchymal marker and miR-204-3p as an epithelial marker. Finally, we demonstrated that miR-196b-3p and miR-204-3p in serum exosomes were differentially expressed among intraductal papillary mucinous neoplasms, mucinous cystic neoplasms, and PC. Conclusion: Serum exo-miRNA biomarkers potentially identify the pancreatic tumor status through less-invasive methods.
\end{abstract}

Pancreatic cancer (PC), comprising mainly pancreatic ductal adenocarcinoma, is one of the deadliest malignancies worldwide and the seventh leading cause of cancer-related mortality with an estimated 432,000 deaths in 2018 (1).

This article is freely accessible online.

Correspondence to: Suguru Yamada, MD, Ph.D., Department of Gastroenterological Surgery (Surgery II), Nagoya University Graduate School of Medicine, 65, Tsurumai-cho, Showa-ku, Nagoya, 466-8550, Japan. Tel: +81 527442245, Fax: +81 527442255, e-mail: suguru@med.nagoya-u.ac.jp

Key Words: Epithelial-mesenchymal transition, pancreatic cancer, exosomes, micro RNA.
PC's poor clinical prognosis is generally attributed to the high frequency of local invasiveness and distant metastasis (2). Epithelial-to-mesenchymal transition (EMT) has important roles in cancer cell invasion and metastasis (3). We previously reported that the EMT status of PC, as defined by immunohistochemistry for E-cadherin and vimentin, was associated with $\mathrm{PC}$ prognosis and the mesenchymal type had higher invasive features such as peritoneal invasion, portal vein invasion, and lymph node metastasis (4). For these reasons, we hypothesized that the clinical behavior of PC, whether locally invasive or metastatic, would be related to the EMT status and the understanding of the EMT status of PC can contribute to development of a novel therapy for PC patients.

Micro RNAs (miRNAs) have been identified as important regulators of gene expression in tissue-specific physiological pathways, in response to environmental cues and in various diseases including human malignancies $(5,6)$. The mature form of miRNAs consist of 21 to 25 base pairs and is capable of inhibiting transcription by inducing degradation of the target mRNAs (5). Although the functional roles of miRNAs in tumor biology are incredibly complicated and all their functions have not been fully understood, we expect that circulating blood miRNA could predict the clinical behavior of PC.

Exosomes are cell-derived microvesicles that are present in different kinds of fluids such as blood, saliva, urine, and also in the culture medium of cell lines (7). Accumulating evidence supports that cancer exosomes play an important role in cell-to-cell communication leading to cancer progression (8). In addition, as exosomes released from cancer cells contain several RNA types including miRNAs, exosomal miRNA (exo-miRNA) could reflect the miRNA signature of the parental cancer cell and can thus inform us on the future behavior of cancer cells (9).

The aim of this study was to identify the exo-miRNA profiles related to the EMT status of PC patients through genome-wide microarray technology. In addition, we attempted to clarify the clinical implication of the selected 
Table I. The TaqMan ${ }^{\circledR}$ assays used in this study.

\begin{tabular}{lccc}
\hline Assay name & AssayID & miRBase (v21) accession number & Mature miRNA sequence \\
\hline hsa-miR-196b* & $121219 \_m a t$ & MIMAT0009201 & UCGACAGCACGACACUGCCUUC \\
hsa-miR-636 & 002088 & MI0003651 & UGUGCUUGCUCGUCCCGCCCGCA \\
hsa-miR-204-3p & $463101 \_m a t$ & MIMAT0022693 & GCUGGGAAGGCAAAGGGACGU \\
hsa-miR-3648 & 464401 mat & MIMAT0018068 & AGCCGCGGGGAUCGCCGAGGG \\
hsa-miR-16 & 000391 & MI0000070 & UAGCAGCACGUAAAUAUUGGCG \\
\hline
\end{tabular}

exo-miRNA biomarkers in clinical specimens derived from patients. Through this approach, we aimed to gain a deeper understanding of the relationship between EMT status and PC that could lead to personalized medicine for PC patients.

\section{Materials and Methods}

Cell lines used in the study. PC cell lines (PANC-1, MIA PaCa-2, HPAF-II, Capan-1, Capan-2, BxPC-3, COLO357, CFPAC-1, AsPC1, SW1990, TU-8902, KP1-NL, and MPanc96) used in this study were purchased from the American Type Culture Collection (Manassas, VA, USA). Cells were maintained in DMEM or RPMI1640 medium (Sigma-Aldrich, St. Louis, MO, USA) supplemented with fetal bovine serum (Thermo Fisher Scientific, Waltham, MA, USA), $100 \mathrm{U} / \mathrm{ml}$ penicillin and $100 \mu \mathrm{g} / \mathrm{ml}$ streptomycin (Life Technologies Corp., Grand Island, NY, USA). All cells were cultured at $37^{\circ} \mathrm{C}$ in a humidified atmosphere of $5 \% \mathrm{CO}_{2}$. EMT status of each PC cell line was determined according to our previous report (4).

Patients and sample collection. This study and all procedures were approved by the Institutional Review Board at Nagoya University and all patients provided written informed consent. All clinical investigations were conducted in accordance with the principles of the Declaration of Helsinki (10). The pancreatic tissues were collected from 16 PC patients undergoing pancreatectomy at the Department of Gastroenterological Surgery, Nagoya University Hospital. All surgically obtained tissue samples mounted in O.C.T. compound (Sakura Finetek, Tokyo, Japan) were immediately frozen in the liquid nitrogen and stored at $-80^{\circ} \mathrm{C}$ until further analysis. We collected serum samples from patients with PC $(n=44)$, intraductal papillary neoplasm (IPMN, $\mathrm{n}=15$ ), and mucinous cystic neoplasm (MCN, $n=4)$, from which we extracted the circulating exosomes

Isolation of exosomes from culture media and nanoparticle tracking analysis. Exosomes were isolated from the culture media of four PC cell lines. To remove cells, collected culture media were centrifuged for $5 \mathrm{~min}$ at $900 \times \mathrm{g}$, followed by another centrifugation for $1 \mathrm{~h}$ at $10,000 \times g$ to remove remaining debris. To remove all particles larger than $200 \mathrm{~nm}$, the centrifuged medium was filtered through $0.2 \mu \mathrm{m}$ pore filters (Sigma-Aldrich, St. Louis, MO, USA). To concentrate the exosomes, the filtrate was passed through a Vivacell 100 (Sartorius AG, Goettingen, Germany) during a $30 \mathrm{~min}$ centrifugation at $4^{\circ} \mathrm{C}$ and $400 \times g$. Ten microliters of the concentrated supernatant were passed through a Sepharose CL-2B (GE Healthcare, Munich, Germany) and $1 \mathrm{ml}$ fractions were eluted. The number of nanoparticles in whole culture-media-derived exosomes was measured using NanoSight NS300 nanoparticle characterization system (NanoSight Ltd., Amesbury, UK). Samples were diluted to $1 / 2000$ and injected into the $405 \mathrm{~nm}$ laser chamber with a constant output controlled by a syringe pump. Three recordings were performed for each sample. Nanoparticle Tracking Analysis software (NanoSight Ltd., Amesbury, UK) was used to measure the size and the concentration of nanoparticles. The Batch Process included in the software was used to integrate the three technical measurements of each sample.

Comprehensive analysis of exo-miRNA expression. To identify an exo-miRNA signature for discriminating the EMT status of PC, comprehensive miRNA- expression profiles of exosomes in culture media from two epithelial cell types and two mesenchymal cell types included interrogation of 2,565 probes, using the 3D-Gene ${ }^{\circledR}$ Human miRNA Oligo Chip ver.21 (TORAY, Kanagawa, Japan). For extraction of exosomes, four cell lines, PANC-1, BxPC-3, MIA $\mathrm{PaCa}-2$, and Capan-2, were cultured and culture medium from each cell line was collected. Total RNA was extracted from the resuspended exosomes using 3D-Gene ${ }^{\circledR}$ RNA extraction reagent from a liquid sample kit (TORAY, Kanagawa, Japan). Comprehensive miRNA expression analysis was performed using a 3D-Gene ${ }^{\circledR}$ miRNA Labeling kit (TORAY, Kanagawa, Japan) and a 3D-Gene ${ }^{\circledR}$ Human miRNA Oligo Chip (TORAY, Kanagawa, Japan) as previously reported (11). All microarray data of the study were in agreement with the Minimum Information About a Microarray Experiment (MIAME) guidelines (12).

Publicly available dataset. Normalized Illumina-HiSeq data for pancreatic adenocarcinoma from The Cancer Genome Atlas (TCGA) were downloaded from the Broad GDAC Firehose (http://gdac.broadinstitute.org/, accessed on June 1 ${ }^{\text {st }}$, 2018). This dataset includes 185 PC cases, including 138 cases with information on recurrence-free survival (RFS). This dataset also includes the sequencing data of four solid normal pancreatic tissues. We also downloaded the normalized microarray data of GSE24279 from Gene Expression Omnibus (https://www.ncbi.nlm.nih.gov/geo/, accessed on June 1st, 2018) for selecting candidate exo-miRNAs (13).

Extraction of exosomes and exo-miRNA from serum specimen. To isolate exosomes from the patients' sera, MagCapture ${ }^{\mathrm{TM}}$ Exosome Isolation Kit PS (Wako, Osaka, Japan) was used according to the manufacturer's instructions. Subsequently, total RNA was extracted from collected exosomes with the use of the microRNA Extractor SP Kit (Wako, Osaka, Japan) according to the manufacturer's instructions.

qPCR analysis. For miRNA-based quantitative PCR (qPCR) analysis, $10 \mathrm{ng}$ of total RNA was reverse transcribed using the 
TaqMan ${ }^{\circledR}$ Micro RNA Reverse Transcription Kit (Applied Biosystems, Foster City, CA, USA) in a total reaction volume of 15 $\mu 1$. qPCR was performed with MicroRNA Assay Kits (Applied Biosystems, Foster City, CA, USA) and TaqMan ${ }^{\circledR}$ Universal MasterMix II, no UNG (Applied Biosystems, Foster City, CA, USA) as previously reported (11). The mean $\mathrm{Ct}$ values of each sample were determined from duplicate reactions. The relative expression level of each miRNA examined was expressed as $\Delta \mathrm{Ct}$, which was defined as the subtraction of the $\mathrm{Ct}$ value of the target miRNA from the Ct value of the internal control miRNA-16 (14). The TaqMan ${ }^{\circledR}$ Assays used in the study are shown in Table I.

Statistical analysis. Continuous variables were compared using Wilcoxon signed-rank test or student $t$-test, as appropriate. Categorical variables were compared using the $\chi^{2}$ or Fisher's exact tests, as appropriate. RFS rates were estimated using the Kaplan-Meier method and compared using a log-rank test. All statistical analyses were performed using R version 3.4.3 (https://www.r-project.org/) and GraphPad Prism 7.03 (Graph Pad Software, San Diego, CA, USA).

\section{Results}

Exosomes collected from the culture media of PC cell lines. To investigate differentially expressed exo-miRNAs between the mesenchymal type of PC and the epithelial type, we first analyzed using microarrays the exo-miRNA expression profile in the culture media of four PC cell lines: PANC-1, BxPC-3, MIA PaCa-2, and Capan-2. Based on the expression levels of E-cadherin and vimentin, PANC-1 and MIA PaCa-2 comprised the mesenchymal cell type, while BxPC-3 and Capan-2 the epithelial cell type. From the collected culture media of each cell line, exosomes were purified using ultracentrifugation and the morphology was confirmed by electron microscopy (Figure 1A). The sizes of the extracellular vesicles including the exosomes were also measured with the nanoparticle tracking analysis and particles with diameters from 30 to $200 \mathrm{~nm}$ were regarded as exosomes (Figure 1B).

Genome-wide analysis of exo-miRNA from the culture media of PC cell lines. As a result of comprehensive exo-miRNA profiling using microarray technology, a heatmap of the differentially expressed exo-miRNAs between mesenchymal and epithelial PC cell types was generated (Figure 2A) (absolute $\log 2$ fold change $>0.58$ ). Of the total of 2,565 miRNAs, 67 miRNAs were expressed at higher levels in exosomes from the mesenchymal type than from the epithelial type $\left[\log _{2}\right.$ fold change (mesenchymal/epithelial) $>0.58$, Figure $2 \mathrm{~B}]$. To filter out the miRNAs from non-cancerous cells, to reduce the number of target miRNAs and, therefore, to increase their practical future utility in the clinic, the microarray data of healthy pancreatic tissue $(n=22)$ and pancreatic ductal adenocarcinoma tissue $(n=136)$ from GSE24279 was also analyzed. There were 193 highly expressed miRNAs in the PC tissues compared with the normal pancreatic tissues in this dataset. Consequently, we selected the two miRNAs, miR-196b-3p and miR-636, in the intersection of 67 miRNAs from our microarray analysis and the 193 miRNAs from GSE24279 (Figure 2B). In addition, with the analysis of our microarray data, three down-regulated exo-miRNAs in the mesenchymal type, miR-204-3p, miR3648 , and miR-4497, were identified by the following criteria: $\log 2$ fold change (mesenchymal/ epithelial) $<2$ and $p<0.05$ derived from student $t$-test (Figure 2C). Finally, we selected five miRNAs for further analyses. To understand the putative function of these five miRNAs, mirPath v.3 analysis was performed that revealed the pathways related to miR-204-3p and miR-3648, but no pathways were associated with the other three miRNAs (Figure 2D) (15).

Analysis with publicly available datasets. As the exosomes related to cancer cell communication are mainly released by cancer cells or surrounding cancer associated cells such as fibroblasts (16), we analyzed the expression of selected miRNAs in PC tissues using the TCGA dataset. Because expression levels of the mature type of selected miRNAs were basically low and the purpose of this study was to elucidate exo-miRNAs related to the EMT status of PC, we downloaded pre-miRNA sequence data from Broad GDAC FIREHOSE instead of mature miRNA sequence data. Based on the normalized RNA-sequencing data of TCGA, four miRNAs except for mir-4497 were available for further analyses. The comparisons between PC tissue and solid normal tissue in the TCGA dataset revealed that the expression levels of mir-196b in PC tissues were relatively higher than those in normal tissues and the expression levels of mir-204 in PC tissues were lower than those in normal tissues (mir-196b, $p=0.18$; mir$204, p=0.33$, Figure 3A). Furthermore, PC cases were divided into two groups according to each miRNA expression levels in PC tissues (Figure 3B). As a result, the RFS of the PC cases with high mir-196b expression was significantly worse than that of PC cases with low mir-196b expression ( $p=0.0003$, Figure 3B) and the RFS of the PC cases with low mir-204 expression was significantly worse than that of PC cases with high mir-204 expression ( $p=0.0014$, Figure 3B).

Expression of selected miRNAs in PC cell lines. Before using specimens derived from patients, we confirmed the expression levels of the identified miRNAs in seven PC cell lines: HPAF-II, Capan-1, Capan-2, BxPC-3, COLO357, CFPAC-1, and AsPC-1. The expression levels of E-cadherin and Vimentin in each cell line are based on our previous study (4). The comparisons of the normalized miRNA expression levels among PC cell lines are provided in Figure 4. Collectively, miR-196b-3p was regarded as a mesenchymal marker and miR-204-3p as an epithelial marker.

Confirmation of the expression levels of selected miRNAs in $P C$ tissue and patient's serum. To investigate the selected 
A
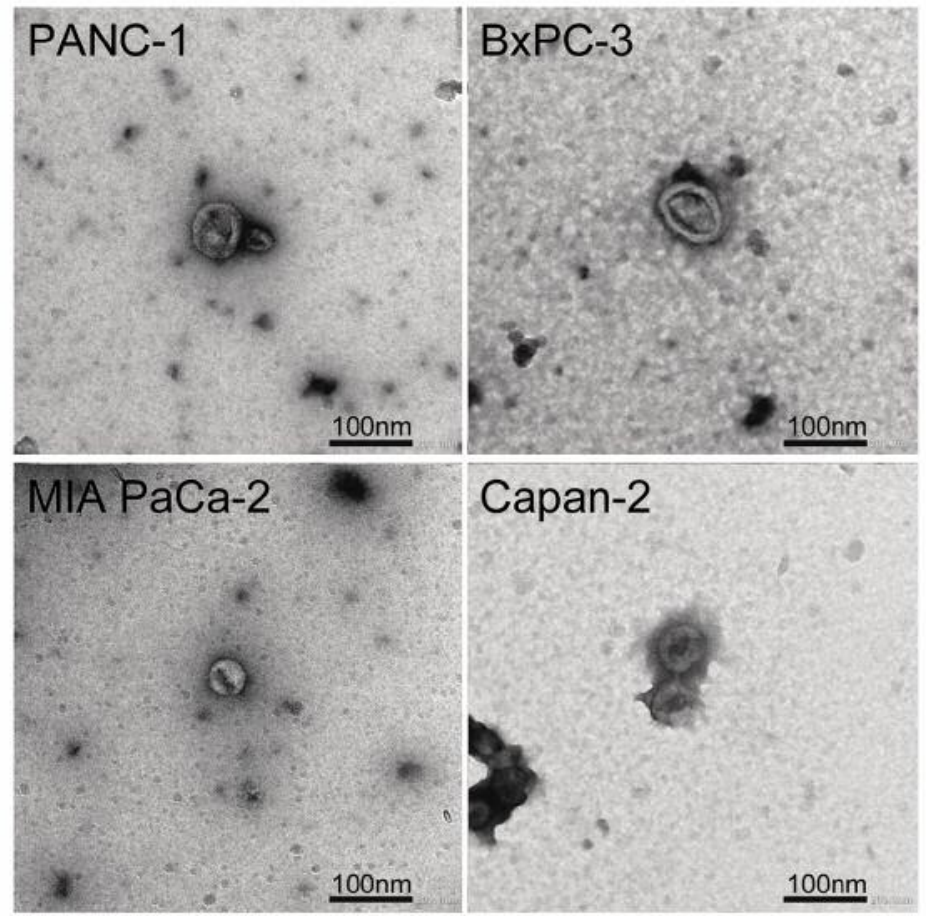

Capan-2

\section{B}
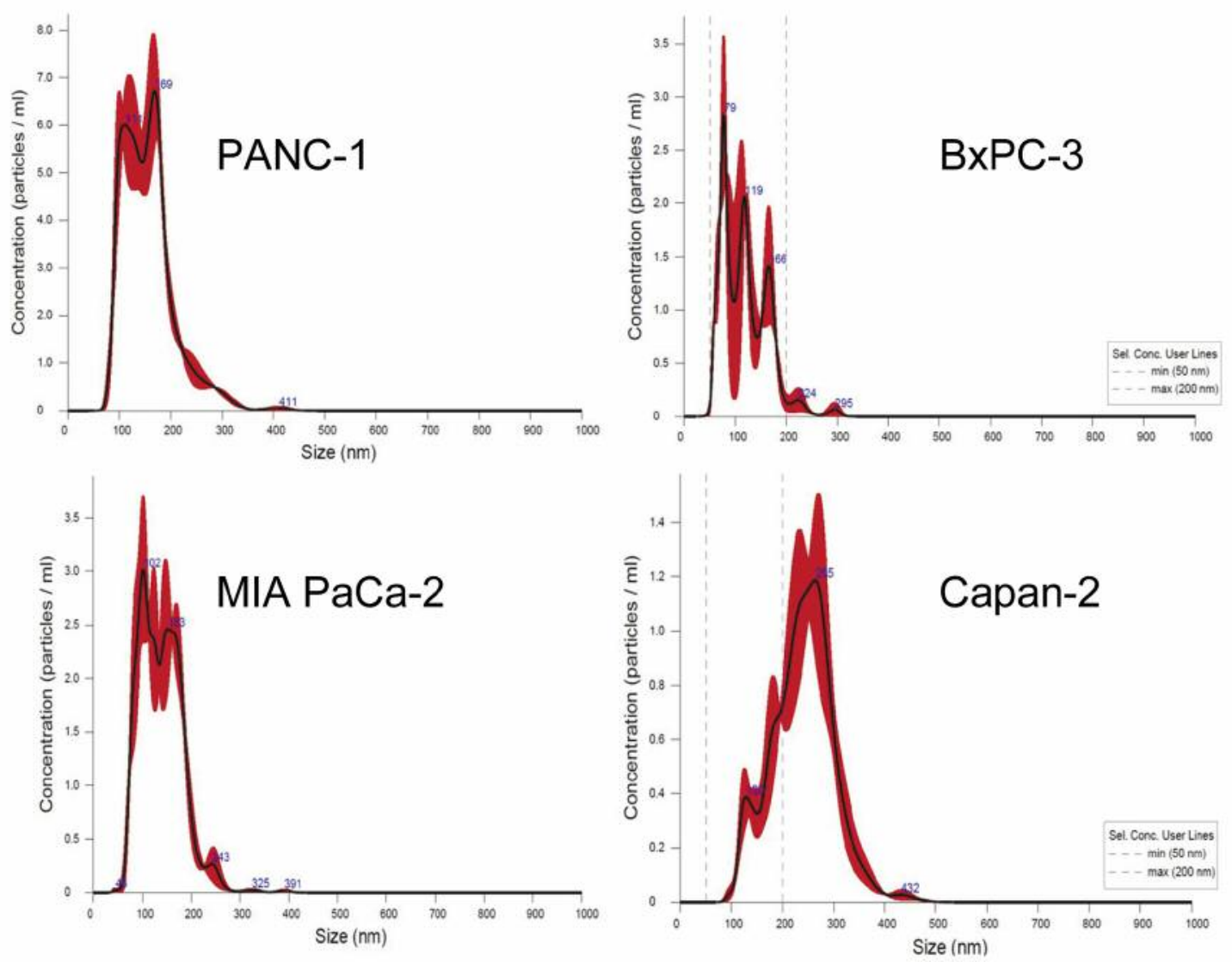

Figure 1. Isolation of exosomes from culture media of pancreatic cancer (PC) cell lines and confirmation with nanoparticle tracking analysis. (A) Images of exosomes captured by an electron microscopy. Exosomes were purified with an ultracentrifuge method from culture media of four PC cell lines. (B) Nanoparticle tracking analysis of extracellular vesicles collected from the culture media of four PC cell lines using the NanoSight NS300 nanoparticle system (NanoSight Ltd., Amesbury, UK). 


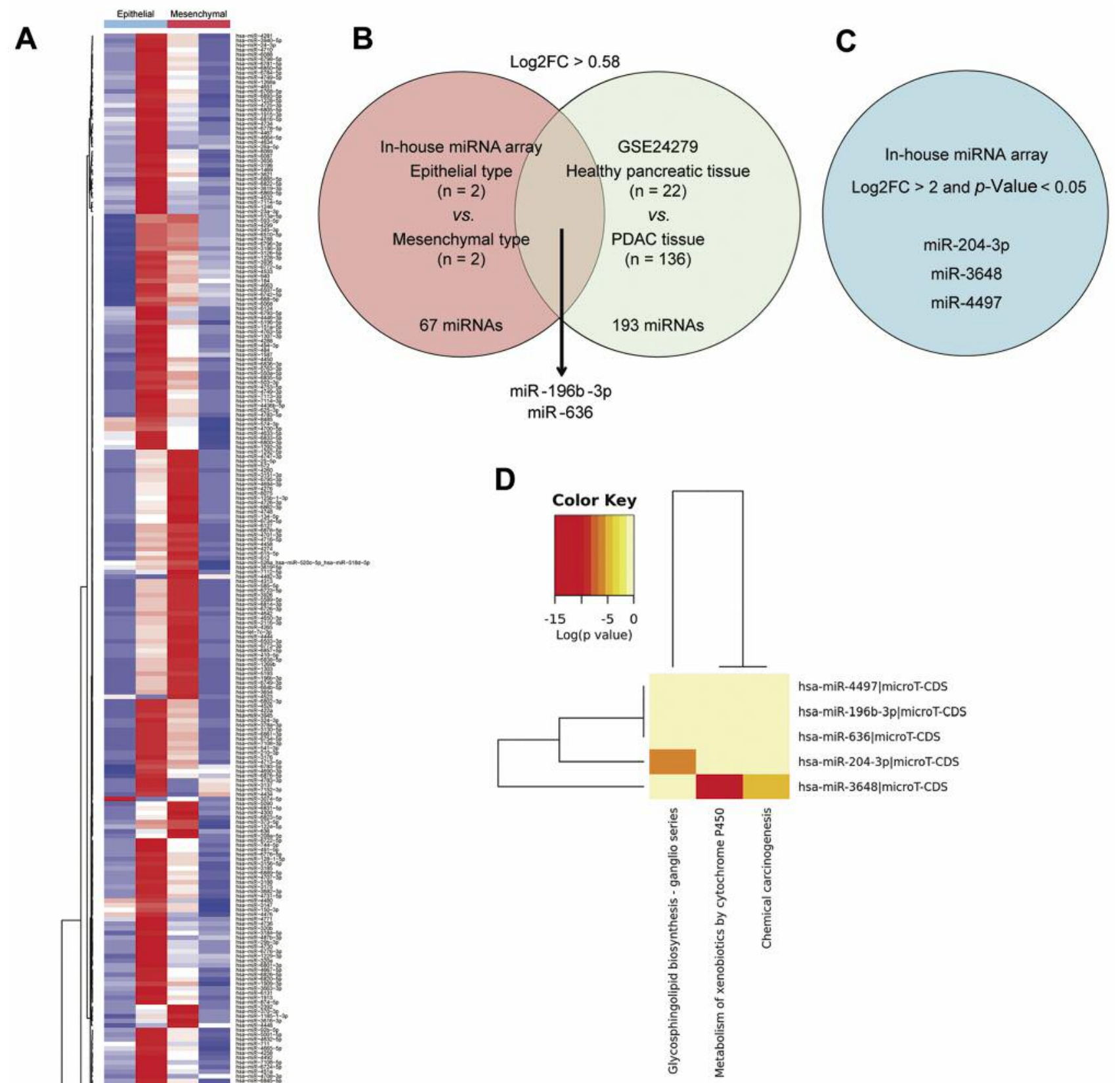

Figure 2. Identification of exosomal micro RNAs (exo-miRNAs) associated with epithelial to mesenchymal transition in pancreatic cancer $(P C)$ cell lines. (A) A heatmap of the differentially expressed exo-miRNAs between mesenchymal and epithelial PC cell lines. There were 291 miRNAs differentially expressed between the two types of PC cells. (B) A Benn diagram of exomiRNAs highly expressed in the mesenchymal type from in-house microarray data and miRNAs highly expressed in pancreatic ductal adenocarcinoma from publicly available microarray dataset (GSE24279). MiR-196b-3p and miR-636 were in the intersection of the two circles. (C) Exo-miRNAs were expressed less in the culture media of mesenchymal PC cell lines. p-Value was derived from student's t-test. (D) Analysis of the putative pathways related to identified miRNAs differentially expressed between exosomes of epithelial and mesenchymal types. 
A mir-196b

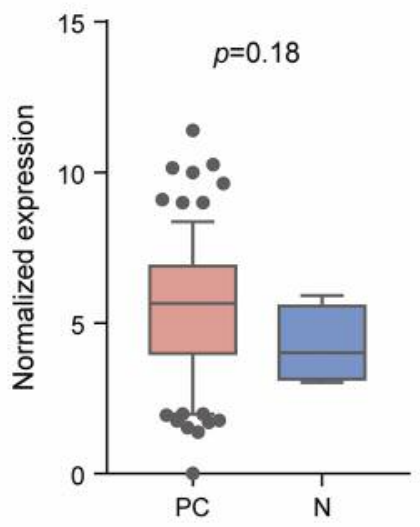

mir-636

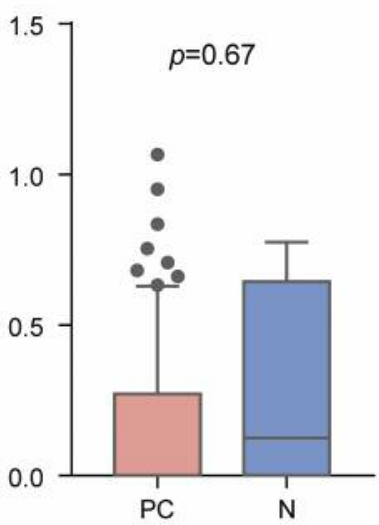

mir-204

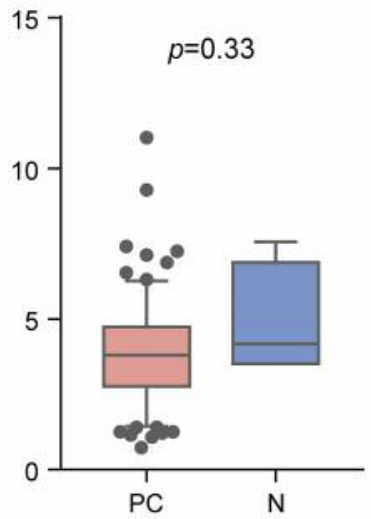

mir-3648

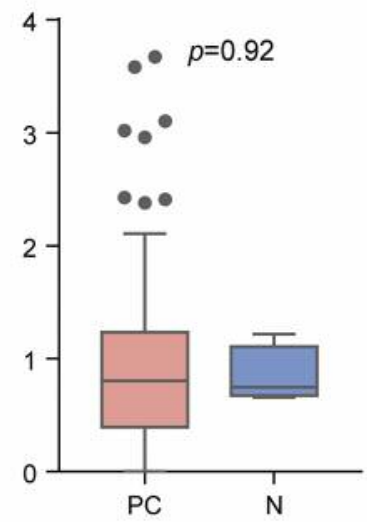

$\mathbf{B}$
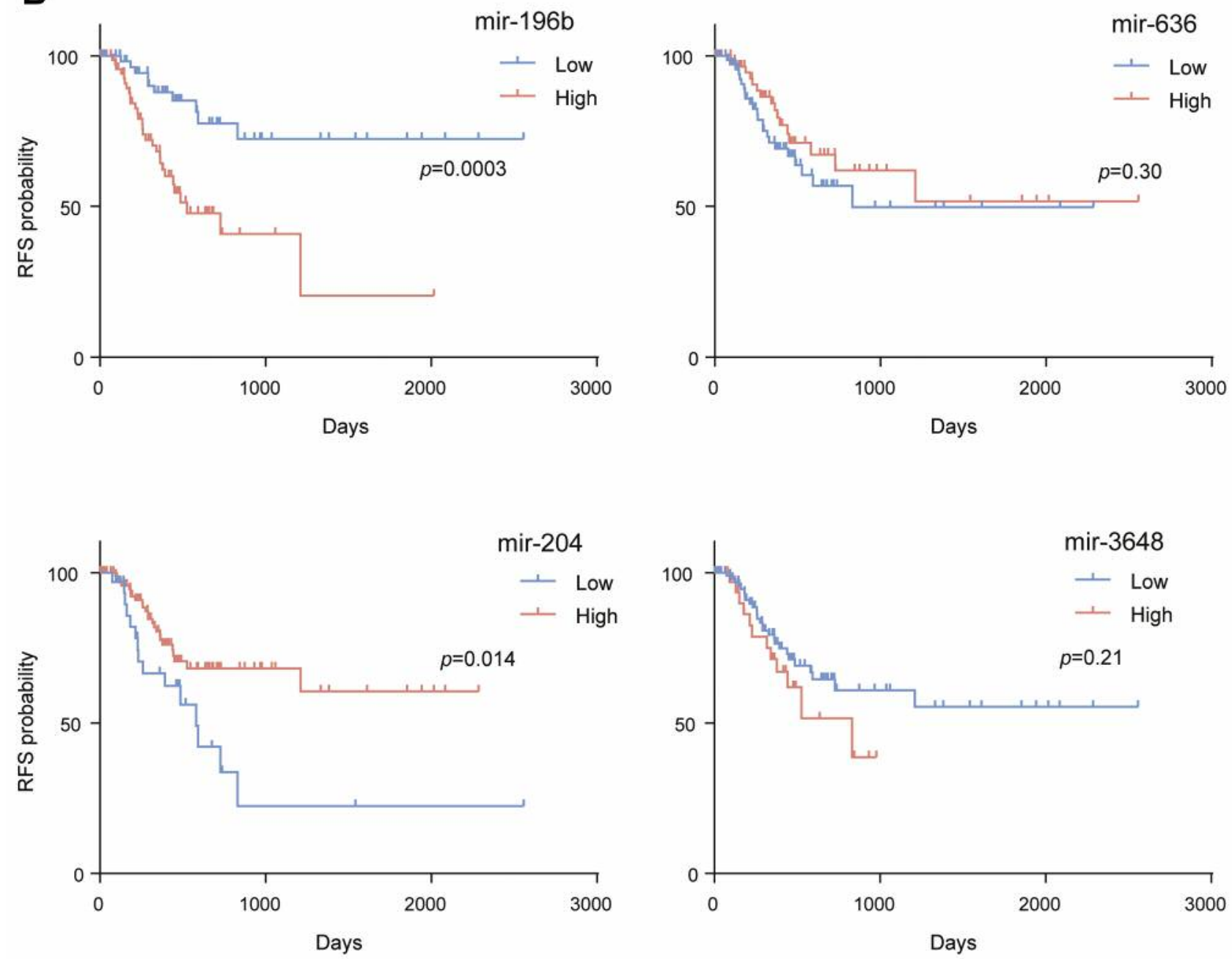

Figure 3. Analysis of identified miRNAs using The Cancer Genome Atlas (TCGA) dataset. (A) Comparisons of expression levels of the selected miRNAs between pancreatic cancer (PC) tissue and non-cancerous pancreatic tissue. Sequencing data of mir-4497 was not available in this dataset. (B) Recurrence free survival (RFS) of PC patients in TCGA stratified by the expression levels of the selected miRNAs. 
A

miR-196b-3p

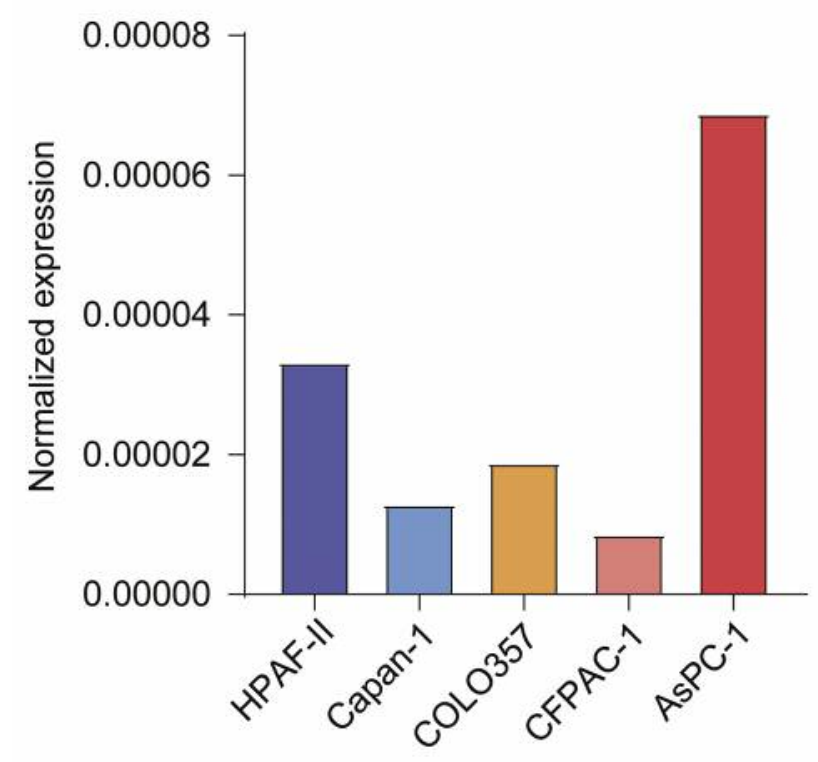

C

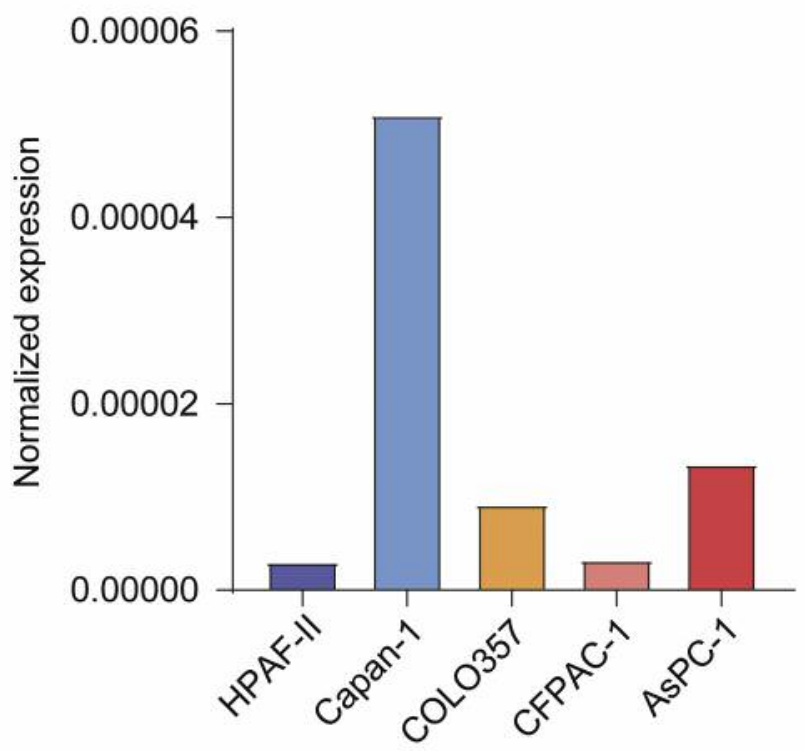

B $\operatorname{miR}-636$

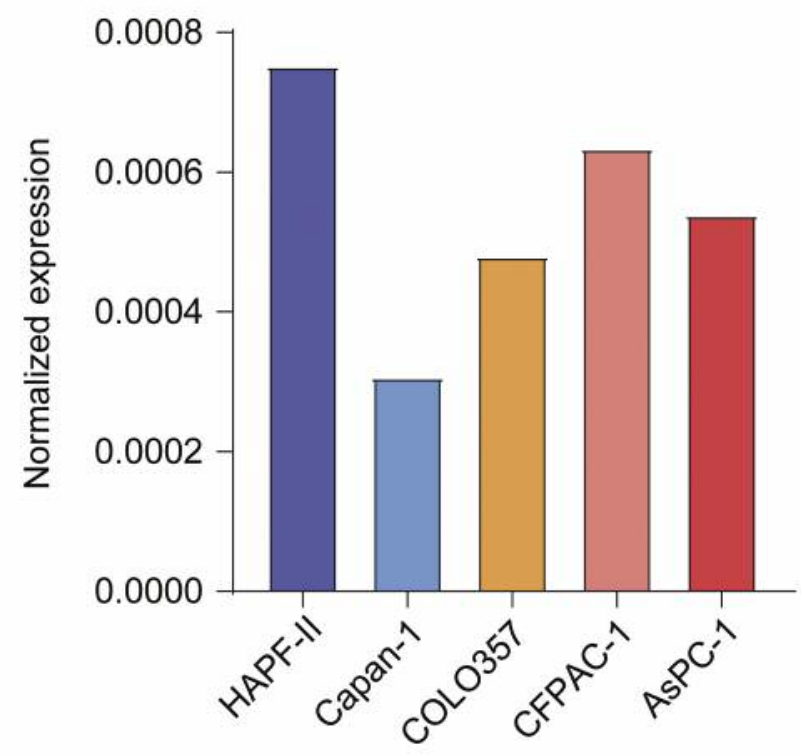

D

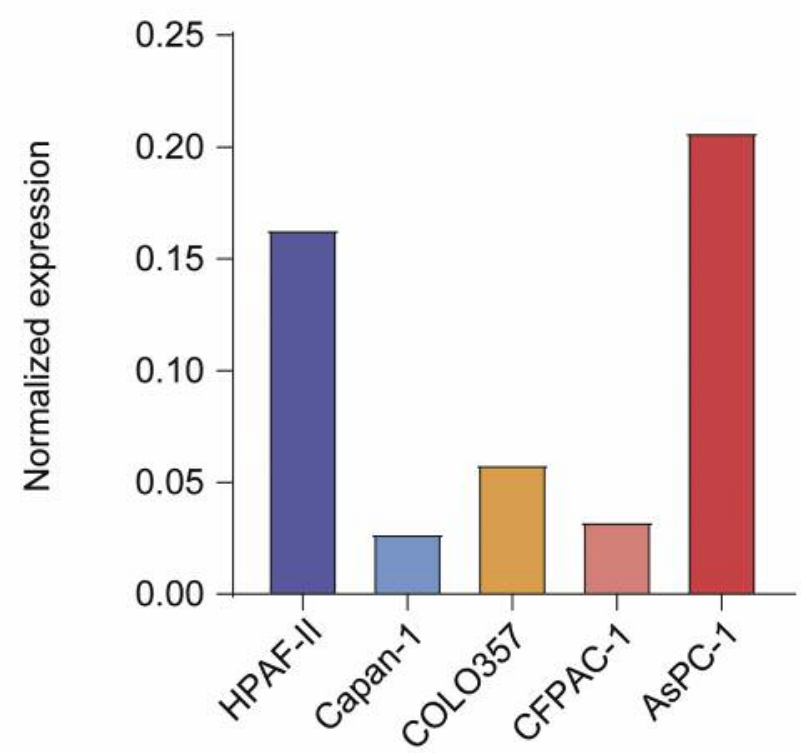

Figure 4. Expression analysis of identified miRNAs in pancreatic cancer cell lines. Expression analysis of identified miRNAs: miR-196b-3p, miR636, and miR-204-3p was performed by quantitative PCR. (A) Normalized expression levels of miR-196b-3p. (B) Normalized expression levels of miR-636. (C) Normalized expression levels of miR-204-3p. (D) Normalized expression levels of miR-3648.

epithelial and mesenchymal exo-miRNA markers in clinical specimens, we extracted total RNA from resected PC tissues embedded with the O.C.T. compound and compared the expression levels in PC tissue and matched adjacent normal tissue. The expression levels of miR-196b-3p were significantly higher in PC tissue than in the paired normal pancreatic tissue ( $p=0.009$, Figure $5 \mathrm{~A}$ ), while the expression levels of miR-204-3p did not differ significantly $(p=0.85$, Figure 5A). In addition, to evaluate the potential utility of these two exo-miRNAs as less-invasive biomarkers, exo-miRNAs in the sera from patients with PC, IPMN, and MCN were extracted and used for quantification of exosomal miR-196b- 
A

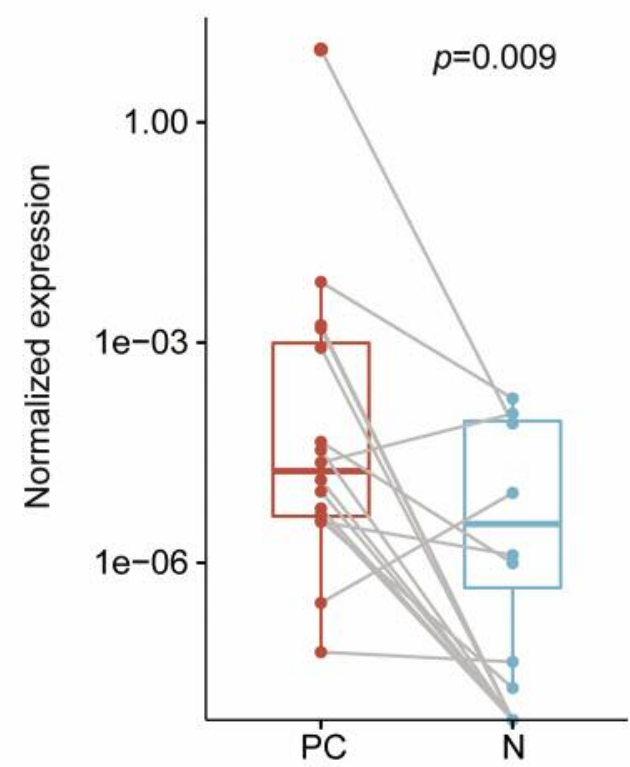

miR-204-3p

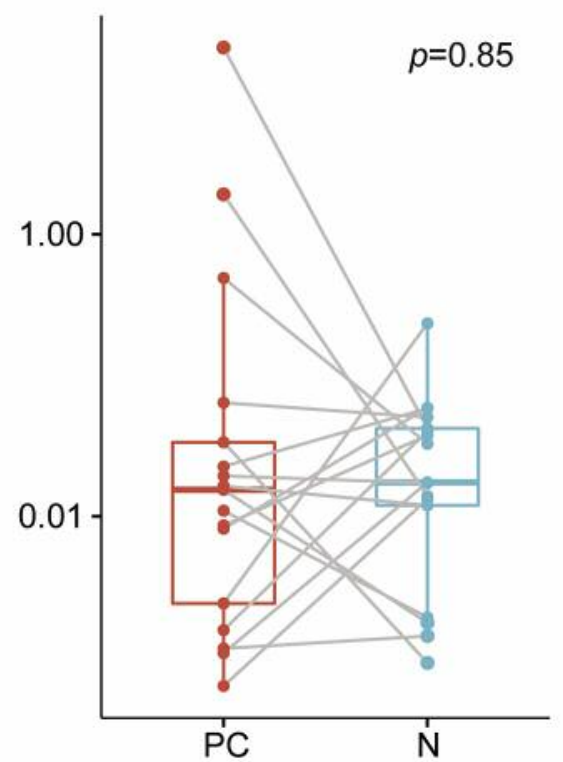

B

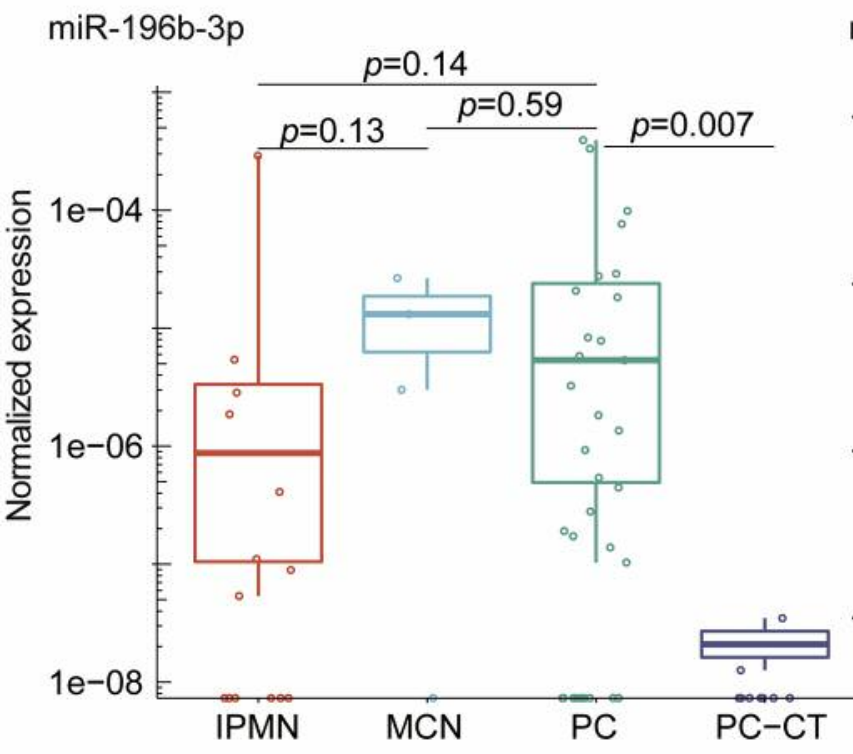

miR-204-3p

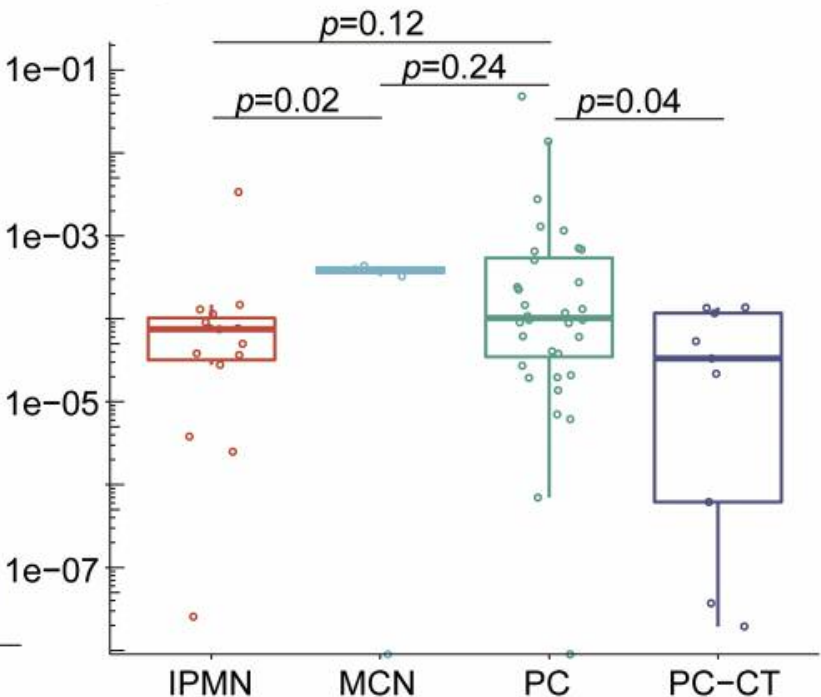

Figure 5. Analysis of miR196b-3p and miR-204-3p in clinical samples. (A) Normalized expression of miR-196b-3p and miR-204-3p in resected pancreatic cancer tissues and matched normal pancreatic tissues. (B) Normalized expression of miR-196b-3p and miR-204-3p in serum specimens derived from patients with intraductal papillary mucinous neoplasm, mucinous cystic neoplasm, and pancreatic cancer. PC: Pancreatic cancer; N: normal pancreatic tissue; IPMN: interpapillary mucinous neoplasm; MCN: mucinous cystic neoplasm; PC-CT: pancreatic cancer treated with chemotherapy.

$3 p$ and miR-204-3p. Expression of miR-196b-3p in serum exosomes -from PC patients was higher than that in IPMN patients with marginal significance ( $p=0.14$, Figure 5B, while there was no significant difference between $\mathrm{MCN}$ and $\mathrm{PC}$ patients ( $p=0.59$, Figure 5B). The expression of miR-204-3p was significantly higher in exosomes derived from the serum of MCN patients than that from IPMN patients $(p=0.02$, $p=0.007$, Figure 5B), while there was marginally significant difference between MCN and PC patients ( $p=0.24$, Figure 5B). Furthermore, the comparison between serum exosomes from 
non-treated PC patients and PC patients treated with chemotherapy indicated that both exo-miRNAs were significantly lower in PC patients treated with chemotherapy (miR-196b-3p, $p=0.007$; miR-204-3p, $p=0.04$, Figure 5B).

\section{Discussion}

In this study, we performed a comprehensive miRNA microarray-based expression profiling analysis of culture media from four different PC cell lines, to identify an exomiRNA biomarker for the EMT status of an original PC cell. We subsequently evaluated the expression of selected miRNAs in publicly available datasets and PC cell lines to understand the significance of the expression levels of these miRNAs in PC tissues and cells. Finally, we demonstrated that miR-196b-3p and miR-204-3p were differentially expressed in serum exosomes from IPMN, MCN, and PC. Namely, serum exo-miRNA biomarkers potentially characterize the pancreatic tumor status through less-invasive methods.

$\mathrm{PC}$ is one of the lethal diseases due to the fact that it easily spreads through the perineural spaces surrounding abdominal arteries $(17,18)$. In addition, the pancreas is anatomically juxtaposed to arteries such as the superior mesenteric artery, the hepatic artery, and the celiac artery and poses a difficulty in removing the locally advanced PC with sufficient resection margins. While some PCs extensively invade surrounding tissues through the perineural space, other PCs are diagnosed as completely resectable, and distant metastasis such as liver and peritoneal metastasis occurs after resection. Thus, advanced PCs comprise different types of $\mathrm{PC}$, and have a different progression behavior.

EMT, the capability of cells to switch between epithelial and mesenchymal type, has been essential for the generation of complex body patterns and is the mechanism used by cancer cells to disperse throughout the body (19). We have previously reported that the EMT status is one of the independent prognostic factors for surgically resected PCs. The expression of lysyl oxidase homolog 2 (LOXL2), one of the important regulators of EMT, was significantly related to PC patients' survival and it is one of the putative therapeutic targets $(4,20)$. In addition, evidence supports that EMT may mediate chemoresistance in PC and defining the EMT status of each PC patient might contribute to suitable multidisciplinary therapy $(21,22)$. However, clinically useful biomarkers for distinguishing the EMT status of PC have not been identified.

Cancer cells secrete various types of humoral factors. Exosomes are small membranous vesicles that differ in their cellular origin and contain mRNA, DNA, proteins, and miRNA (23). Extracellular vesicles including exosomes and microvesicles from cancer cells have been found in the blood of cancer patients and therefore, provide a source of cancerrelated molecules (24). In addition, several studies have shown that exo-miRNAs promote metastases and enhance endothelial cell migration $(25,26)$, and cancer-associated fibroblasts can also secret exosomes, which can regulate the survival and proliferation of cancer cells (16). Therefore, as exosomal molecules can inform us about the type and nature of the original tumor cell, we analyzed the expression of exo-miRNAs using a microarray-based technology.

In the current study, miR-196b-3p and miR-636 were found to be upregulated in the exosomes from mesenchymal PC cells. MiR-196b-3p is a human myometrial miRNA that is modulated by exogenous oxytocin and its expression has been shown to be downregulated in a rat model of lipopolysaccharide-induced acute lung injury $(27,28)$. Park et al. have revealed that the intrinsic constitutive signaling circuit composed of IkB $\alpha / \mathrm{NF}$ $\mathrm{kB}(\mathrm{p} 65)$, miR-196b-3p, Meis2, and PPP3CC is formed during the emergence of castration-resistant prostate cancer and this circuit controls the expression of stem cell transcription factors that drive high tumorigenicity (29). Schultz et al. have reported two microRNA panels in whole blood for the detection of PC using the combination of four or 10 miRNAs including miR636 (30). In another study, miR-636 has also been identified as commonly down regulated miRNA in recurrent prostate cancer compared with non-recurrent prostate cancer by meta-analysis of six publicly available datasets (31). We also examined the expression levels of miR-204-3p in resected PC tissue and sera from patients with IPMN, MCN, and PC. In several kinds of human malignancies, miR-204 acts as tumor suppressor but the role of miR-204 in cancer development remains controversial $(32,33)$. Roldo et al. investigated miRNA expression profiles in normal pancreas tissue and pancreatic tumors including insulinomas, and they found miR-204 is expressed primarily in insulinomas and correlates with immunohistochemical expression of insulin (34). Besides, in the study for discovering a urine biomarker for early detection of PC with microarray analysis, miR-204 showed statistically higher levels in Stage I compared to Stages II, III, and IV (35). In our analysis of serum specimens, the expression of miR-204-3p in $\mathrm{MCN}$ patients was significantly higher than that in IPMN and PC patients. Thus, the expression of miR-204 may be downregulated in advanced PC and may be relatively high in early stage of PC or other low-malignant pancreatic tumors.

The recent advancements in broad genomic and transcriptomic analysis using microarray or high-throughput sequencing procedures have resulted in molecular characterization of several cancer types (36-38). However, to our best knowledge, this study is the first to extract miR-196b-3p and miR-636 from exosomes of PC cells and to identify the association with the EMT status of PC. Although, our results indicate that the discovered exo-miRNAs are differentially expressed among exosomes derived from IPMN, MCN, and PC, there were some inherent limitations to this study. First, in biomarker discovery, we should consider the potential difference between cell lines and heterogeneous human tissue. We employed four PC cell lines to elucidate differentially expressed miRNAs in exosomes 
from epithelial and mesenchymal cell types because the heterogeneity of the clinical specimens diminishes the difference between the two types. Second, the clinical specimens were very limited and from a single institution. To confirm the practical utility of the discovered biomarkers, evaluation of prospectively corrected specimens should be conducted. Finally, as we cannot analyze the relation between the selected exo-miRNAs and clinical features such as chemo-sensitivity and survival, we are now collecting blood samples from PC patients undergoing neoadjuvant chemotherapy, adjuvant chemotherapy, and chemotherapy for unresectable or metastatic PCs for a future analysis of the identified exo-miRNA biomarkers.

\section{Conclusion}

In conclusion, we identified novel exo-miRNAs associated with the EMT status of PC using genome-wide exo-miRNA profiling in exosomes from the culture media of PC cell lines. Our findings may contribute in the identification of less-invasive biomarkers for EMT status of PC patients and improve the decision for PC therapy.

\section{Conflicts of Interest}

The Authors declare no potential conflicts of interest associated with this manuscript.

\section{Authors' Contributions}

Conception and design: FS; SY, Financial support: SY; YK, Administrative support: YK, Provision of study materials and patients: HT; MT; MK; CT; DK; GN; MK; MF, Collection and assembly of data: FS; ST; MH; MS; YS, Microarray data analysis: ST; FS, Public data analysis: FS, qPCR data analysis and interpretation: FS; MH; MS; YS, Manuscript writing: FS; ST; SY, Final approval of manuscript: all Authors.

\section{Acknowledgements}

The Authors would like to thank Yoko Nishikawa and Yasuko Iguchi for arranging clinical samples and helping to perform the experiments.

\section{Funding}

This work was partially supported by the Japan Society for the Promotion of Science (JSPS) KAKENHI Grant-in-Aid for Scientific Research (C) number 16K10590 to Suguru Yamada. All funders had no role in the design of the study and collection, analysis, and interpretation of data and in writing the manuscript.

\section{References}

1 Ferlay J, Ervik M, Lam F, Colombet M, Mery L, Piñeros M, Znaor A, Soerjomataram I and Bray F: Global Cancer Observatory: Cancer Today. Lyon, France: International Agency for Research on Cancer. Available from: https://gco.iarc.fr/today, accessed on $20^{\text {th }}$ January 2020.
2 Stathis A and Moore MJ: Advanced pancreatic carcinoma: Current treatment and future challenges. Nat Rev Clin Oncol 7(3): 163172, 2010. PMID: 20101258. DOI: 10.1038/nrclinonc.2009.236

3 De Craene B and Berx G: Regulatory networks defining EMT during cancer initiation and progression. Nat Rev Cancer 13(2): 97-110, 2013. PMID: 23344542. DOI: $10.1038 /$ nrc3447

4 Yamada S, Fuchs BC, Fujii T, Shimoyama Y, Sugimoto H, Nomoto S, Takeda S, Tanabe KK, Kodera Y and Nakao A: Epithelial-to-mesenchymal transition predicts prognosis of pancreatic cancer. Surgery 154(5): 946-954, 2013. PMID: 24075276. DOI: 10.1016/j.surg.2013.05.004

5 Brennecke J, Hipfner DR, Stark A, Russell RB and Cohen SM: Bantam encodes a developmentally regulated microrna that controls cell proliferation and regulates the proapoptotic gene hid in drosophila. Cell 113(1): 25-36, 2003. PMID: 12679032. DOI: 10.1016/s0092-8674(03)00231-9

6 Schickel R, Boyerinas B, Park SM and Peter ME: Micrornas: Key players in the immune system, differentiation, tumorigenesis and cell death. Oncogene 27(45): 5959-5974, 2008. PMID: 18836476. DOI: 10.1038/onc.2008.274

7 Siravegna G, Marsoni S, Siena S and Bardelli A: Integrating liquid biopsies into the management of cancer. Nat Rev Clin Oncol 14(9): 531-548, 2017. PMID: 28252003. DOI: 10.1038/nrclinonc.2017.14

8 Valadi H, Ekstrom K, Bossios A, Sjostrand M, Lee JJ and Lotvall JO: Exosome-mediated transfer of mrnas and micrornas is a novel mechanism of genetic exchange between cells. Nat Cell Biol 9(6): 654-U672, 2007. PMID: 17486113. DOI: 10.1038/ncb1596

9 Zhang J, Li S, Li L, Li M, Guo CY, Yao J and Mi SL: Exosome and exosomal microrna: Trafficking, sorting, and function. Genomics Proteomics Bioinformatics 13(1): 17-24, 2015. PMID: 25724326. DOI: $10.1016 /$ j.gpb.2015.02.001

10 World Med A: World medical association declaration of helsinki ethical principles for medical research involving human subjects. JAMA 310(20): 2191-2194, 2013. PMID: 24141714. DOI: 10.1001/jama.2013.281053

11 Niwa Y, Yamada S, Sonohara F, Kurimoto K, Hayashi M, Tashiro M, Iwata N, Kanda M, Tanaka C, Kobayashi D, Nakayama G, Koike M, Fujiwara M and Kodera Y: Identification of a serumbased mirna signature for response of esophageal squamous cell carcinoma to neoadjuvant chemotherapy. J Transl Med 17(1): 1, 2019. PMID: 30602370. DOI: 10.1186/s12967-018-1762-6

12 Brazma A, Hingamp P, Quackenbush J, Sherlock G, Spellman P, Stoeckert C, Aach J, Ansorge W, Ball CA, Causton HC, Gaasterland T, Glenisson P, Holstege FC, Kim IF, Markowitz V, Matese JC, Parkinson H, Robinson A, Sarkans U, SchulzeKremer S, Stewart J, Taylor R, Vilo J and Vingron M: Minimum information about a microarray experiment (miame)-toward standards for microarray data. Nat Genet 29(4): 365-371, 2001. PMID: 11726920 . DOI: $10.1038 / n g 1201-365$

13 Bauer AS, Keller A, Costello E, Greenhalf W, Bier M, Borries A, Beier M, Neoptolemos J, Buchler M, Werner J, Giese N and Hoheisel JD: Diagnosis of pancreatic ductal adenocarcinoma and chronic pancreatitis by measurement of microrna abundance in blood and tissue. PLoS One 7(4): e34151, 2012. PMID: 22511932. DOI: 10.1371/journal.pone.0034151

14 Song J, Bai Z, Han W, Zhang J, Meng H, Bi J, Ma X, Han S and Zhang Z: Identification of suitable reference genes for qpcr analysis of serum microrna in gastric cancer patients. Dig Dis Sci 57(4): 897-904, 2012. PMID: 22198701. DOI: 10.1007/s10620011-1981-7 
15 Vlachos IS, Zagganas K, Paraskevopoulou MD, Georgakilas G, Karagkouni D, Vergoulis T, Dalamagas T and Hatzigeorgiou AG: Diana-mirpath v3.0: Deciphering microrna function with experimental support. Nucleic Acids Res 43(W1): W460-466, 2015. PMID: 25977294. DOI: 10.1093/nar/gkv403

16 Richards KE, Zeleniak AE, Fishel ML, Wu J, Littlepage LE and Hill R: Cancer-associated fibroblast exosomes regulate survival and proliferation of pancreatic cancer cells. Oncogene 36(13): 1770-1778, 2017. PMID: 27669441. DOI: 10.1038/onc.2016.353

17 Liebl F, Demir IE, Mayer K, Schuster T, D'Haese JG, Becker K, Langer R, Bergmann F, Wang K, Rosenberg R, Novotny AR, Feith M, Reim D, Friess H and Ceyhan GO: The impact of neural invasion severity in gastrointestinal malignancies a clinicopathological study. Ann Surg 260(5): 900-908, 2014 PMID: 25379860. DOI: 10.1097/SLA.0000000000000968

18 Kayahara M, Nakagawara H, Kitagawa $\mathrm{H}$ and Ohta T: The nature of neural invasion by pancreatic cancer. Pancreas 35(3): 218-223, 2007. PMID: 17895841. DOI: 10.1097/mpa.0b013e3180619677

19 Acloque H, Adams MS, Fishwick K, Bronner-Fraser M and Nieto MA: Epithelial-mesenchymal transitions: The importance of changing cell state in development and disease. J Clin Invest 119(6): 1438-1449, 2009. PMID: 19487820. DOI: 10.1172/JCI38019

20 Tanaka N, Yamada S, Sonohara F, Suenaga M, Hayashi M, Takami H, Niwa Y, Hattori N, Iwata N, Kanda M, Tanaka C, Kobayashi D, Nakayama G, Koike M, Fujiwara M, Fujii T and Kodera Y: Clinical implications of lysyl oxidase-like protein 2 expression in pancreatic cancer. Sci Rep 8(1): 9846, 2018. PMID: 29959362. DOI: 10.1038/s41598-018-28253-9

21 Arumugam T, Ramachandran V, Fournier KF, Wang HM, Marquis L, Abbruzzese JL, Gallick GE, Logsdon CD, McConkey DJ and Choi W: Epithelial to mesenchymal transition contributes to drug resistance in pancreatic cancer. Cancer Res 69(14): 5820-5828, 2009. PMID: 19584296. DOI: 10.1158/00 08-5472.CAN-08-2819

22 Zheng XF, Carstens JL, Kim J, Scheible M, Kaye J, Sugimoto $\mathrm{H}$, $\mathrm{Wu} \mathrm{CC}$, LeBleu VS and Kalluri R: Epithelial-tomesenchymal transition is dispensable for metastasis but induces chemoresistance in pancreatic cancer. Nature 527(7579): 525530, 2015. PMID: 26560028. DOI: 10.1038/nature 16064

23 Rahbari M, Rahbari N, Reissfelder C, Weitz J and Kahlert C: Exosomes: Novel implications in diagnosis and treatment of gastrointestinal cancer. Langenbecks Arch Surg 401(8): 10971110, 2016. PMID: 26560028. DOI: 10.1038/nature16064

24 Xu R, Rai A, Chen M, Suwakulsiri W, Greening DW and Simpson RJ: Extracellular vesicles in cancer - implications for future improvements in cancer care. Nat Rev Clin Oncol, 2018. PMID: 29795272. DOI: 10.1038/s41571-018-0036-9

25 Fang JH, Zhang ZJ, Shang LR, Luo YW, Lin YF, Yuan Y and Zhuang SM: Hepatoma cell- secreted exosomal microrna-103 increases vascular permeability and promotes metastasis by targeting junction proteins. Hepatology, 2018. PMID: 29637568. DOI: $10.1002 /$ hep. 29920

26 Valencia K, Luis-Ravelo D, Bovy N, Anton I, Martinez-Canarias S, Zandueta C, Ormazabal C, Struman I, Tabruyn S, Rebmann V, De Las Rivas J, Guruceaga E, Bandres E and Lecanda F: Mirna cargo within exosome-like vesicle transfer influences metastatic bone colonization. Mol Oncol 8(3): 689-703, 2014. PMID: 24593875. DOI: 10.1016/j.molonc.2014.01.012

27 Cook JR, MacIntyre DA, Samara E, Kim SH, Singh N, Johnson MR, Bennett PR and Terzidou V: Exogenous oxytocin modulates human myometrial micrornas. Am J Obstet Gynecol 213(1): 65.e6169, 2015. PMID: 25757635. DOI: 10.1016/j.ajog.2015.03.015

28 Park J, Jeong S, Park K, Yang K and Shin S: Expression profile of micrornas following bone marrow-derived mesenchymal stem cell treatment in lipopolysaccharide-induced acute lung injury. Exp Ther Med 15(6): 5495-5502, 2018. PMID: 29904430. DOI: 10.3892/etm.2018.6118

29 Jeong JH, Park SJ, Dickinson SI and Luo JL: A constitutive intrinsic inflammatory signaling circuit composed of mir-196b, meis2, ppp3cc, and p65 drives prostate cancer castration resistance. Mol Cell 65(1): 154-167, 2017. PMID: 28041912. DOI: $10.1016 /$ j.molcel.2016.11.034

30 Schultz NA, Dehlendorff C, Jensen BV, Bjerregaard JK, Nielsen KR, Bojesen SE, Calatayud D, Nielsen SE, Yilmaz M, Hollander $\mathrm{NH}$, Andersen KK and Johansen JS: Microrna biomarkers in whole blood for detection of pancreatic cancer. JAMA 311(4): 392-404, 2014. PMID: 24449318. DOI: 10.1001/jama.2013.284664

31 Pashaei E, Pashaei E, Ahmady M, Ozen M and Aydin N: Metaanalysis of mirna expression profiles for prostate cancer recurrence following radical prostatectomy. PLoS One 12(6): e0179543, 2017. PMID: 28651018. DOI: 10.1371/journal.pone.0179543

32 Mikhaylova O, Stratton Y, Hall D, Kellner E, Ehmer B, Drew AF, Gallo CA, Plas DR, Biesiada J, Meller J and CzyzykKrzeska MF: Vhl-regulated mir-204 suppresses tumor growth through inhibition of lc3b-mediated autophagy in renal clear cell carcinoma. Cancer Cell 21(4): 532-546, 2012. PMID: 22516261. DOI: $10.1016 /$ j.ccr.2012.02.019

33 Lee H, Lee S, Bae H, Kang HS and Kim SJ: Genome-wide identification of target genes for mir-204 and mir-211 identifies their proliferation stimulatory role in breast cancer cells. Sci Rep 6: 25287, 2016. PMID: 27121770. DOI: 10.1038/srep25287

34 Roldo C, Missiaglia E, Hagan JP, Falconi M, Capelli P, Bersani S, Calin GA, Volinia S, Liu CG, Scarpa A and Croce CM: Microrna expression abnormalities in pancreatic endocrine and acinar tumors are associated with distinctive pathologic features and clinical behavior. J Clin Oncol 24(29): 4677-4684, 2006. PMID: 16966691. DOI: 10.1200/JCO.2005.05.5194

35 Debernardi S, Massat NJ, Radon TP, Sangaralingam A, Banissi A, Ennis DP, Dowe T, Chelala C, Pereira SP, Kocher HM, Young BD, Bond-Smith G, Hutchins $\mathrm{R}$ and Crnogorac-Jurcevic T: Noninvasive urinary mirna biomarkers for early detection of pancreatic adenocarcinoma. Am J Cancer Res 5(11): 3455-3466, 2015. PMID: 26807325.

36 Cancer Genome Atlas Research Network: Comprehensive genomic characterization defines human glioblastoma genes and core pathways. Nature 455(7216): 1061-1068, 2008. PMID: 18772890. DOI: $10.1038 /$ nature07385

37 Liu CG, Calin GA, Volinia S and Croce CM: Microrna expression profiling using microarrays. Nat Protoc 3(4): 563578, 2008. PMID: 18388938. DOI: 10.1038/nprot.2008.14

38 Sonohara F, Gao F, Iwata N, Kanda M, Koike M, Takahashi N, Yamada Y, Kodera Y, Wang X and Goel A: Genome-wide discovery of a novel gene-expression signature for the identification of lymph node metastasis in esophageal squamous cell carcinoma. Ann Surg, 2017. PMID: 29240008. DOI: 10.1097/SLA.0000000000002622

Received February 23, 2020

Revised March 7, 2020

Accepted March 9, 2020 